\title{
Time in the eye of the beholder: Gaze position reveals spatial-temporal associations during encoding and memory retrieval of future and past
}

\author{
Corinna S. Martarelli ${ }^{1,2}$ - Fred W. Mast ${ }^{1,2}$ - Matthias Hartmann ${ }^{1,2}$
}

Published online: 21 July 2016

(C) Psychonomic Society, Inc. 2016

\begin{abstract}
Time is grounded in various ways, and previous studies point to a "mental time line" with past associated with the left, and future with the right side. In this study, we investigated whether spontaneous eye movements on a blank screen would follow a mental timeline during encoding, free recall, and recognition of past and future items. In all three stages of processing, gaze position was more rightward during future items compared to past items. Moreover, horizontal gaze position during encoding predicted horizontal gaze position during free recall and recognition. We conclude that mental time line and the stored gaze position during encoding assist memory retrieval of past versus future items. Our findings highlight the spatial nature of temporal representations.
\end{abstract}

Keywords Mental time line $\cdot$ Encoding $\cdot$ Free recall ·

Recognition · Eye movements

\section{Introduction}

Humans can revisit the past and anticipate the future. Remembering the past and imagining the future seem to involve similar cognitive and neuronal processes (e.g., De Vito \& Della Sala, 2011; Schacter, Addis, \& Buckner, 2008). A growing body of evidence suggests that time is structured in terms of space (e.g., Bonato, Zorzi, \& Umiltà, 2012; Winter,

Corinna S. Martarelli

corinna.martarelli@psy.unibe.ch

1 Department of Psychology, University of Bern, Fabrikstrasse 8, CH-3012 Bern, Switzerland

2 Center for Cognition, Learning and Memory, University of Bern, Bern, Switzerland
Marghetis, \& Matlock, 2015). In order to conceptualize the mental representation of time via a spatial frame of reference, different authors have suggested a "mental time line" (Barsalou, 2008; Boroditsky, 2000; Casasanto \& Boroditsky, 2008; Stocker, 2012). In cultures with a left-to-right reading habit, the mental time line is mapped over a horizontal axis, with past events on the left and future events on the right side (e.g., Casasanto \& Jasmin, 2012; Flumini \& Santiago, 2013; Hartmann, Martarelli, Mast, \& Stocker, 2014; Ishihara, Keller, Rossetti, \& Prinz, 2008; Ouellet, Santiago, Funes, \& Lupiáñez, 2010; Santiago, Lupiáñez, Pérez, \& Funes, 2007; Torralbo, Santiago, Lupiáñez, 2006; Weger \& Pratt, 2008).

Here we aim to further investigate the role of the horizontal spatial frame of reference in temporal cognition by means of eye movements. Eye movements are principally used to extract knowledge from the visual environment. However, it has been shown that eye movements also accompany mental processes in the absence of visual stimuli. For example, when listening to a description of a house, the listener's gaze position on a blank screen moves up when the description proceeds from the ground floor to higher floors, showing that internal inspection of a mental image triggers eye movements in the external visual space (Spivey \& Geng, 2001). Interestingly, eye movements on a blank screen are not only triggered by internal inspection of concrete mental images (e.g., a house), but also by more conceptual spatial structures such as the mental number line (Hartmann, Mast, \& Fischer, 2015, 2016; Loetscher, Bockisch, Nicholls, \& Brugger, 2010) or the mental time line (Hartmann et al., 2014; Stocker, Hartmann, Martarelli, \& Mast, 2015). These results can be interpreted in favor of the embodied cognition approach, according to which knowledge and meaning is grounded in sensorimotor experiences, and bodily activities constantly contribute to cognition (e.g., Barsalou, 2008; Glenberg, Witt, \& Metcalfe, 2013). As such, eye movements might be used to 
"act out" the spatial relation according to which mental images (Spivey \& Geng, 2001) or knowledge is represented.

A current question of interest is when does time associate with space (e.g., Tillman, Tulagan, \& Barner, 2015; Winter et al., 2015). Does the mental time line lead to an immediate structuring of incoming past and future related information with the left and right representational space, or does this association only emerge when information is retrieved from memory? In order to address this question, it is necessary to look at spatial-temporal associations during different stages of memory. A promising way to investigate spatial-temporal associations in different memory processes has recently been introduced by Saj, Fuhrman, Vuilleumier, and Boroditsky (2014). In their study, participants had to encode, recall, and recognize a list of images as belonging to the past or future. They tested patients suffering from left hemispatial neglect and could demonstrate that these patients not only neglect the left side of space but also past events. This finding highlighted that temporal associations are grounded in space and space seems to play a functional role for the understanding of time.

In this study we aimed to extend Saj et al.'s approach to a non-pathological context by investigating whether healthy people use their eyes to encode, recall, and recognize temporal events in a mental time line congruent manner. Based on Saj et al.'s results it is not possible to conclude the involvement of a mental time line during encoding. Spatial-temporal associations exist in temporal categorization tasks where past and future-related items (such as past and future verbs) need to be classified as past or future (e.g., Santiago et al., 2007). This suggests that the mental time line leads to an immediate spatial structuring of incoming past and future-related information. Thus it is possible that eye gaze position will reflect a past-left and future-right association already during encoding. In addition, the mental time line might be recruited to structure information in memory, and consequently influence information processing at a later stage, when the information is retrieved from memory (Saj et al., 2014). In a mental time travel task, we found that eye gaze shifted more rightward when participants thought about their personal future than when they thought about their past (Hartmann et al., 2014), thus we expect that eye gaze position will reflect a past-left and future-right association also during recall and recognition in this study.

The comparison of spatial-temporal associations during different memory processes (encoding, recall, recognition) will provide insights about when a spatial reference frame, such as the mental time line, structures cognition. In addition, this study will also contribute to the understanding of memory-driven eye movements more generally. Previous research showed that eye movements are involved in the retrieval of visual information. For example, it has been found that eye position during memory retrieval (in the absence of the remembered objects) is similar to eye position during encoding, suggesting that spatial information is stored as part of the memory representation (e.g., Johansson, Holsanova, \& Holmqvist, 2006; Martarelli \& Mast, 2011; 2013; Martarelli, Chiquet, Laeng, \& Mast, 2016; Richardson \& Spivey, 2000; Richardson \& Kirkham, 2004; Spivey \& Geng, 2001; Wantz, Martarelli, \& Mast, 2016). Moreover, it has been shown that returning the eyes to spatial locations where the stimuli were previously encoded facilitates memory retrieval (Johansson, Holsanova, Dewhurst, \& Holmqvist, 2012; Johansson \& Johansson, 2014; Laeng \& Teodorescu, 2002; Laeng, Bloem, D’Ascenzo, \& Tommasi, 2014; Scholz, Mehlborn, \& Krems, 2016). However, previous research investigating the "looking at nothing phenomenon" always presented visual information during encoding. For the first time in the study presented here we have a blank screen both during encoding and during retrieval. Will the eyes of our participants during retrieval reenact the spatial position during encoding? Thus, eye movements may play two different roles during the task adopted in this study: First, they may follow the spatial association of the to-be-remembered item category (future, past), and secondly they may also be determined by the stored gaze position during encoding (regardless of the past-left and future-right association). By using both the gaze position during encoding and the left-right association of the items as predictors, our data will allow us to assess the weight of these two mechanisms in determining eye movements during memory retrieval.

To summarize, we aimed to analyze and compare the impact of a mental time line during encoding, free recall, and recognition of future and past, and to assess whether eye movements during retrieval processes were determined by spatial-temporal associations over and above the eye movement pattern during encoding.

\section{Method}

\section{Participants}

Thirty participants took part in this study (21 women, mean age: 21.5 years, range: 19-25 years). Participants gave written informed consent prior to the study. All participants had normal or corrected-to-normal visual acuity and were German speaking.

\section{Apparatus}

Eye movements were recorded by using an SMI RED tracking system (SensoMotoric Instruments, Teltow, Germany). Data were registered with a sampling rate of $50 \mathrm{~Hz}$, a spatial resolution of $0.1^{\circ}$, and a gaze position accuracy of $0.5^{\circ}$. The eyetracking device is contact-free and determines the gaze by 
combining the cornea reflex with the pupil location, via an infrared light sensitive video camera. The stimuli were presented on a 17-in. screen $(1,280 \times 1,024$ pixel $)$ using Experiment Center Software and eye data were recorded with I-View X Software, both developed by SensoMotoric Instruments (SensoMotoric Instruments, Teltow, Germany).

\section{Materials and design}

The task consisted of items that participants needed to encode, recall, and recognize as belonging to the past or future. The items were objects or verbs that a fictive person liked to eat (egg, corn, hamburger, spaghetti, ice cream, apple, grapes, cake, sandwich, and pear), had in his apartment (clock, desk, kettle, vase, telephone, lamp, toaster, refrigerator, chair, and television), liked to wear (shoe, shirt, pants, watch, sock, tie, scarf, boot, belt, and coat), and liked to do (sailing, rollerskating, painting, playing football, playing the guitar, reading, flying a kite, playing tennis, skiing, and playing the trumpet) 10 years ago in the past, or that the same person will like to eat, will have in his apartment, will wear, and will like to do 10 years in the future. We used the same items as Saj et al. (2014), with the difference that we did not use pictures of the items but audiofiles, presented via loudspeakers. We wanted to avoid that eye movement behavior was determined by specific properties of the visual images (e.g., mentally revisiting specific parts of the image during recall and recognition).

Each of the categories (food, objects in apartment, cloths, activities) constituted a separate block with an encoding, free recall, and recognition phase. Within each category, half of the items were associated with past and the other half with future. To associate the items with the past and future, we cued the words of the encoding task with either a low (peak $=452 \mathrm{~Hz}$ ) or a high tone (peak $=887 \mathrm{~Hz}$ ). Participants learned the pitch-time association prior to the encoding phase. Importantly, the association between the items and the categories past and future, as well as the association between the pitch and past and future, was counterbalanced across participants. Each tone lasted $1 \mathrm{~s}$, and the audiofiles lasted on average $698 \mathrm{~ms}(S D=229)$. The auditory material was created by means of Audacity (http://audacity.sourceforge.net). The screen remained gray during the entire experiment, with the exception of the instructions, which appeared on the screen.

\section{Procedure}

The procedure was adapted from Saj et al. (2014). Participants were seated in front of the computer screen. The distance between participants and the screen was approximately 70 $\mathrm{cm}$. We introduced a cover story to conceal the hypothesis of this study. They were told that the study is about the relationship between pupil dilation during different tasks, including memory retrieval. After a 5-point calibration and validation procedure (only error values below $0.8^{\circ}$ were accepted), task instructions appeared on the screen: "You will hear different words. The words are related to the future or to the past. Your task is to encode the words and to remember whether they are related to the future or to the past. Next, you will have to freely recall the words (free recall) and then to recognize the words (old/new discrimination). The experiment consists of four blocks; in each block you are presented with words related to a theme. Words that are preceded by a high tone (resp. low tone) concern the past. Words that are preceded by a low tone (respectivel. high tone) concern the future. Now you will have examples of the tone." Participants then heard two examples of items preceded by a low and high tone (these items were not used in the main study, e.g., sun and rain). Each example was followed by a written feedback repeating the tone-time associations.

Learning of the tone-time association In this phase, participants had to learn the association between the pitch height (low vs. high) and time (past vs. future). Half of the participants were told that the low-pitched tone would indicate an item belonging to the future list, and a high-pitched tone an item belonging to the past list, and vice versa for the other half of participants. They were presented with five low and five high tones in random order. After each tone presentation, they had to say whether the tone indicates past or future. A feedback screen repeating the pitch-time association was presented after each response. All participants correctly learned the tone-time associations.

Encoding As in Saj et al. (2014), participants were introduced to a fictional man named David. Since our participants (students) were younger compared to the participants in Saj et al.'s study (patients), we changed the age of the protagonist. Thus, participants were instructed as follows in the first block: "Today, David is 25 years old. In the first part of this study, you will learn about things that David liked to eat 10 years ago (when he was 15 years old) and things that he will like to eat in 10 years (when he will be 35 years old)". We added: "the things that David liked to eat 10 years ago are preceded by a high tone (as cue for the past or future, respectively) and the things that David will like to eat in 10 years are preceded by a low tone (as cue for the future or past, respectively)". When participants did not have any further questions, the experimenter started the task. Half of the items were cued with past (five items), and the other half with future (association counterbalanced across participants) and the ten items were presented in random order. A trial started with the presentation of the tone, followed by a 1-s silent interval before the item was presented. After the onset of the item, participants had $4 \mathrm{~s}$ to encode and memorize the item until the next trial started. The screen remained gray during the whole task. After participants encoded the ten items, they continued with the free recall task. 
Free recall Participants were instructed to freely recall all of the food items and to say whether it was something "David liked 10 years ago" or "will like in 10 years from now". As soon as participants indicated the temporal association, the experimenter pressed a button to initiate the next recording period (while also noting the response). ${ }^{1}$ The recording periods (consisting of the items and temporal classifications) lasted on average 7,552 $\mathrm{ms}(S D=2324)$. As in the encoding task, the screen remained gray during the whole time. After the free recall task, participants continued to the recognition task.

Recognition Participants heard the ten items of the encoding phase (without tone) and four new items, presented in random order. Participants were instructed to say whether they had heard the item before (old/new discrimination), and if it was something that "David liked 10 years ago" or something he "will like 10 years from now". As in the recall task, the experimenter noted the responses and immediately started the next trial as soon as the participant completed the temporal classification. The screen remained gray and the recording periods lasted on average 4,829 $\mathrm{ms}(S D=1076)$.

The same procedure (encoding, free recall, recognition) was repeated three times more, with things that David had in his apartment (e.g., vase), with things he liked to wear (e.g., boot), and with things he liked to do (e.g., sailing). The written instructions of the encoding, free recall, and recognition tasks were repeated at each block. Finally, participants were asked to guess the hypothesis of the study.

\section{Results}

When asked about the aim of the study, one participant guessed the hypothesis (past-left and future-right associations). Data of this participant were excluded from the analysis. Two more participants were excluded from the analyses, one for technical reasons (the tracking ratio was of $42 \%$ ), and the other person had accuracy values of the memory performance of more than $3 S D$ under the mean of all participants ( $17.5 \%$ correctly remembered items in the free recall task and $12.5 \%$ of correct responses in the recognition task).

The first $2 \mathrm{~s}$ (tone and 1-s silent interval) were excluded from the analyses of the encoding task. Analyses of the free recall and recognition task were based on correct trials regarding both memory performance and temporal categorization performance. Participants were correct in $62.8 \%(S D=$ 14.7) of the trials in the free recall task and in $79.0 \%(S D=$

\footnotetext{
${ }^{1}$ The experimenter simply made a cross for correctly remembered future and past items, so that he had enough resources to press the button to start a new recording period immediately after the temporal classification was completed. Moreover, the experimenter said "ok" after each button press and the participant was instructed to continue with the next item only after the "ok" was given.
}

13.0) of the trials in the recognition task. More specifically, in the free recall task participants correctly remembered $69.4 \%$ $(S D=12.2)$ of the items, and correctly identified the temporal dimension of $91.0 \%(S D=13.7)$ of these items. In the recognition task they correctly identified $95 \%(\mathrm{SD}=5)$ of the items as old and correctly identified the temporal dimension of $84.0 \%(S D=9.6)$ of these trials. Analyses were based on fixations extracted using Be-Gaze software (SensoMotoric Instruments, Teltow, Germany). Fixations were detected when the sum of the gaze stream on the $\mathrm{x}$ - and $\mathrm{y}$-axes was below 100 pixels and when the fixation duration exceeded $80 \mathrm{~ms}$. Blink events were subtracted from the original gaze stream by the software. Trials that lasted more than $10 \mathrm{~s}$ in the free recall task (10.0\% of the trials) and in the recognition task $(1.7 \%$ of the trials) were eliminated. Fixations outside the screen were excluded $(7.5 \%)$. On average participants executed $5.85(S D=$ 1.76) fixations per trial in the encoding task, $8.47(S D=3.01)$ fixations per trial in the free recall task, and $6.17(S D=1.96)$ fixations per trial in the recognition task.

The main analyses focus on the mean horizontal and vertical eye gaze position (dependent variables) for past and future items during the encoding, free recall, and recognition tasks. Thus the independent variables are time (past vs. future) and task (encoding, free recall, recognition). Given that we were also interested in the impact of the gaze position during encoding for the gaze position during retrieval processes, we further included this variable in the analyses. In order to get a more detailed picture of the gaze position during the different tasks we additionally considered the time course for each task.

\section{Position of eye gaze}

We computed the mean eye gaze position on the $\mathrm{x}$-axis (horizontal axis) in pixels for each participant for past versus future items. Zero represents the centre of the screen, positive values represent locations on the right, and negative values represent locations on the left side of the screen. Thus, the larger the values on the $\mathrm{x}$-axis, the more the position of the gaze was on the right part of the screen. We conducted a repeated measures ANOVA with time (past vs. future) and task (encoding, free recall, recognition) as within-subjects factors and mean gaze position on the $\mathrm{X}$-axis as dependent variable. When sphericity was not met (Mauchly's test reached a p-value $<.05$ ) we corrected the degrees of freedom according to Huynh-Feldt. The analysis revealed a significant effect of time, $F(1,26)=7.81, p=.010$, $\eta_{\mathrm{p}}{ }^{2}=0.23$. For past items the mean gaze position was 41 pixels more leftward compared to future items (Past: -51 pixels, $S E M=$ 23, Future: -10 pixels, $S E M=24)$. It has to be noted that despite the fact that participants shifted their gaze more to the left with past items than with future items, in absolute terms (screen coordinates) the gaze position for future items also was on the left part of the screen (Future: -10 pixels, $S E M=24$ ). Even though the effect of task turned out to be significant, $F(2,52)=4.22, p=$ 
$.020, \eta_{\mathrm{p}}{ }^{2}=0.14$, post hoc tests with Bonferroni correction revealed no significant differences $(p s>$.068). Furthermore, the interaction between time and task turned out to be significant by trend, $F(1.66,43.25)=2.64, p=.092, \eta_{\mathrm{p}}{ }^{2}=0.09$. Planned comparisons (simple effects of time within each level of task) show a significant difference between past and future in the encoding task: For future items the mean gaze position was 33 pixels more rightward compared to past items, $F(1,26)=7.49$, $p$ $=.011, \eta_{\mathrm{p}}{ }^{2}=0.22$. In the free recall task, the mean gaze position for future items showed a rightward shift of 61 pixels when compared to past items, $F(1,26)=6.89, p=.014, \eta_{\mathrm{p}}{ }^{2}=0.21$. And finally in the recognition task the mean gaze position for future items was 29 pixels more rightward compared to past items, $F(1,26)=4.27, p=.049, \eta_{\mathrm{p}}{ }^{2}=0.14$. The differences between past and future in the three tasks remain significant after Bonferroni-Holm correction $(p=.011<0.017$; $p=.014<.025$; $p=.049<.05)$. Figure 1 illustrates the mean horizontal gaze position for future and past items in the three tasks.

Given that previous research investigating eye position on the screen during processing of time also found a vertical (up-down) projection of the mental time line (Hartmann et al., 2014; Stocker et al., 2015), we analyzed the vertical position of the eye during the three tasks. The analysis of the mean eye gaze position on the y-axis (vertical axis) revealed no significant result. We computed the same model as before. All ps were > 110 .

\section{Prediction of eye gaze position during retrieval processes by spatial-temporal association and encoding position}

We were interested in the effect of time (past vs. future) on the horizontal eye gaze position during memory retrieval over and above the influence of the horizontal eye gaze position during encoding. Thus, the horizontal eye gaze position during encoding was treated as covariate in the next analyses. First, we computed a linear mixed-effects model with random intercepts for participants, and fixed effects for time (past vs. future) and for the horizontal eye gaze position during encoding. The horizontal eye gaze position during free recall was the dependent variable. The horizontal eye gaze position was computed for each trial by averaging the fixations. The degrees of freedom were obtained by a Satterthwaite approximation (Fai \& Cornelius, 1996) implemented in IBM SPSS Statistics, version 22 . The results revealed a significant effect of time, $F$ (1, $637.62)=6.75, p=.010$. Time was coded as 0 for past and as 1 for future. The estimated linear effect of time was 34.73 ( $S E=13.37, p=.010)$, thus showing that the horizontal eye gaze position was shifted more leftward with past-related items during free recall (see Fig. 1). The analysis also illustrated a significant effect of the horizontal eye gaze position during encoding, $F(1,487.17)=41.54, p<.001$. The estimated linear effect of the horizontal eye gaze position was 0.30 ( $S E=0.05, p$ $<.001$ ), indicating a significant positive relationship between

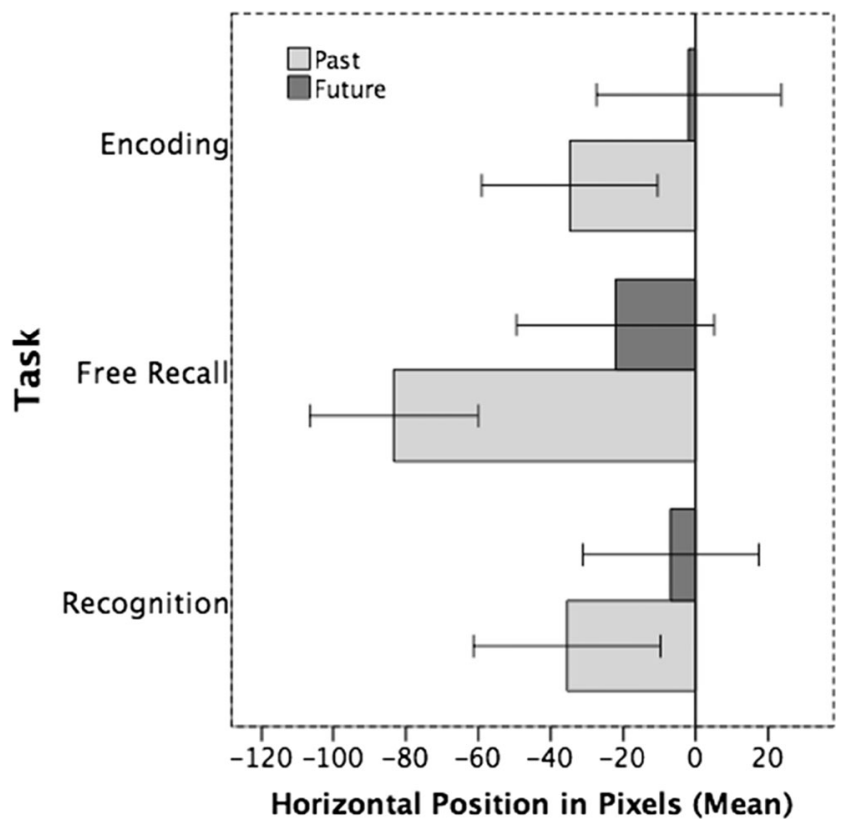

Fig. 1 Mean horizontal eye gaze position (in pixels) for future and past items in the encoding, free recall, and recognition task. The vertical line $(0$ pixels) represents the middle vertical line of the screen. The lower the values on the $\mathrm{x}$-axis, the more to the left was the eye position on the screen. Error bars indicate 1 standard error of the mean

the horizontal eye gaze position during encoding and the horizontal eye gaze position during free recall.

The same linear mixed-effects model was computed for the horizontal eye gaze position during recognition as dependent variable. The results revealed a significant effect of time, $F$ (1, $764.84)=7.55, p=.006$. Time was coded as 0 for past and as 1 for future. The estimated linear effect of time was 28.44 (SE = $10.35, p=.006$ ), thus showing that the horizontal eye gaze position was shifted more leftward with past-related items during recognition (see Fig. 1). The results also illustrated a significant effect of the horizontal eye gaze position during encoding, $F(1,779.53)=11.50, p=.001$. The estimated linear effect of the horizontal eye gaze position was 0.13 ( $S E=0.04, p=.001$ ), thus indicating a significant positive relationship between horizontal eye gaze position during encoding and horizontal eye gaze position during recognition. Taken together, these results illustrate that gaze position during encoding indeed predict gaze position during recall and recognition of that item, and, most importantly, time (past vs. future) still significantly contributed to the prediction of the horizontal eye gaze position after statistically controlling for the position during encoding.

Recent studies have shown serial order effects in the mental number line (e.g., van Dijck \& Fias, 2011), that is, an association between the left side of space and early items on a remembered sequence, and between the right side and late items of a sequence. Rinaldi, Brugger, Bockisch, Bertolini, and Girelli (2015) even found such an effect on eye position during memory recall. In order to further explore the role of serial position on eye movements, we repeated the analysis for memory encoding and 
free recall with the additional predictor serial order (position of the item during encoding). Serial order turned out to be nonsignificant in both analyses $(\mathrm{F}<1)$ and had no impact on the other results. While the absence of an effect of serial order on eye movements during recognition is in line with previous studies (Rinaldi et al., 2015), the absence of such an effect during memory recall is new. However, it is important to note that in Rinaldi et al.'s study, participants were explicitly asked to remember the sequence of the items during encoding, and also to recall the items in the same order as presented during encoding, making the serial position an explicit part of the task and - as a consequence - also an explicit part of the memory representation, which might be a mandatory factor for the serial position effect. In contrast, the serial position was irrelevant in our task, and it is therefore not clear whether participants integrated the serial position information in their memory representation.

Furthermore, we were interested in the relation between the vertical eye gaze position during encoding and the vertical eye gaze position during retrieval. Thus, we computed the same models as before for the vertical eye gaze position. The analyses confirm the absence of an effect of time (past vs. future) on the vertical axis $(F s<1)$, but also show no predicting role of the vertical eye gaze position during encoding for the vertical gaze position both during recall and during recognition ( $p s>.173$ ).

\section{Time-course analysis of eye gaze position}

For all previous analyses, the horizontal gaze position was estimated by averaging the $\mathrm{x}$-coordinates of all fixations of a trial. Here we investigate at what time point of a trial the spatialtemporal association is present. For the encoding task, the horizontal eye gaze position was mapped on a timeline with 100$\mathrm{ms}$ time units. The trials in the free recall and recognition tasks were of different length, depending on participants' responses. In order to compare the time course within and between these two tasks, we performed a time-normalization. All trials were stretched or compressed to a fixed amount of 50 samples through linear interpolation. In all tasks, we compared the gaze position for past and future-related items in each time point. We considered a difference as meaningful when at least five consecutive time points yield a p-value below 0.05 (see Hartmann et al., 2015, for a similar approach). The time windows with significant differences between past and future are represented by the grey area in Fig. 2 .

Importantly, in all three tasks, there was no difference at the beginning of the trial showing that our findings cannot be explained by differences that preexisted before participants encoded, recalled, or recognized the item. In the encoding phase, the lines significantly separate one second after the onset of the word. In the recall and recognition phase, the differences become meaningful around the middle of the trial, which corresponds approximately to the time point when the item is recalled and recognized.
Distribution of horizontal and vertical eye gaze position change

In all previous analysis, we considered horizontal and vertical change in eye gaze position independent from each other. In order to get a more fine-grained view on the eye gaze position change on the horizontal and vertical spatial axis, we transferred each change in eye gaze position into polar coordinates $\left(0-360^{\circ}\right)$ and plotted the distribution cumulatively for all three tasks (see Fig. 3). The time-course analysis revealed that the relevant position changes took place in the first half of the trials. We therefore restricted the analysis to position changes that occurred between 2 and $4 \mathrm{~s}$ during the encoding task, and within the first $50 \%$ of trial duration during the recall and recognition task. Figure 3 reveals that the difference in horizontal eye gaze position between future and past items is driven by leftward and rightward changes that are characterized by less variation along the vertical axis of space. Noteworthy, in addition to "purely" leftward changes in eye gaze position, changes with an additional small downward component (around $15^{\circ}$ ) were also more frequent during past than during future trials, indicating that the past might be represented not only to the left but also slightly more downward (see also Hartmann et al., 2014; Stocker et al., 2015).

\section{Discussion}

The results from this study illustrate that past versus future items elicited specific eye positions: Participants shifted their gaze more to the right with future items than with past items. This effect was found not only during encoding but also during retrieval processes. Thus, the representation of time in memory is associated with the horizontal axis of space, and the encoding and retrieval of future and past events is accompanied by shifts in spatial attention along that axis. Our results indicate that the representation of time is strongly interlinked with the representation of space. We conclude that this association characterizes cognition in general and is not specific to a given stage of memory.

It has been shown that the spatial position is stored in memory along other properties of the stimuli (e.g. Martarelli \& Mast, 2013). Given that participants shifted their gaze more to the left with past items than with future items already in the encoding task, the question arises whether our participants shifted their gaze more to the left with past items during retrieval processes as a reactivation of the whole memory trace, including spatial position during encoding, or whether the mental time line can influence both encoding and retrieval directly. Time (past vs. future) still significantly predicted the horizontal eye gaze position during memory retrieval with the horizontal eye gaze position during encoding included in the models. Thus, it is unlikely that the results we found are solely based on a reactivation process. In line with this view, Hartmann et al. (2014) showed that participants' eye gaze 

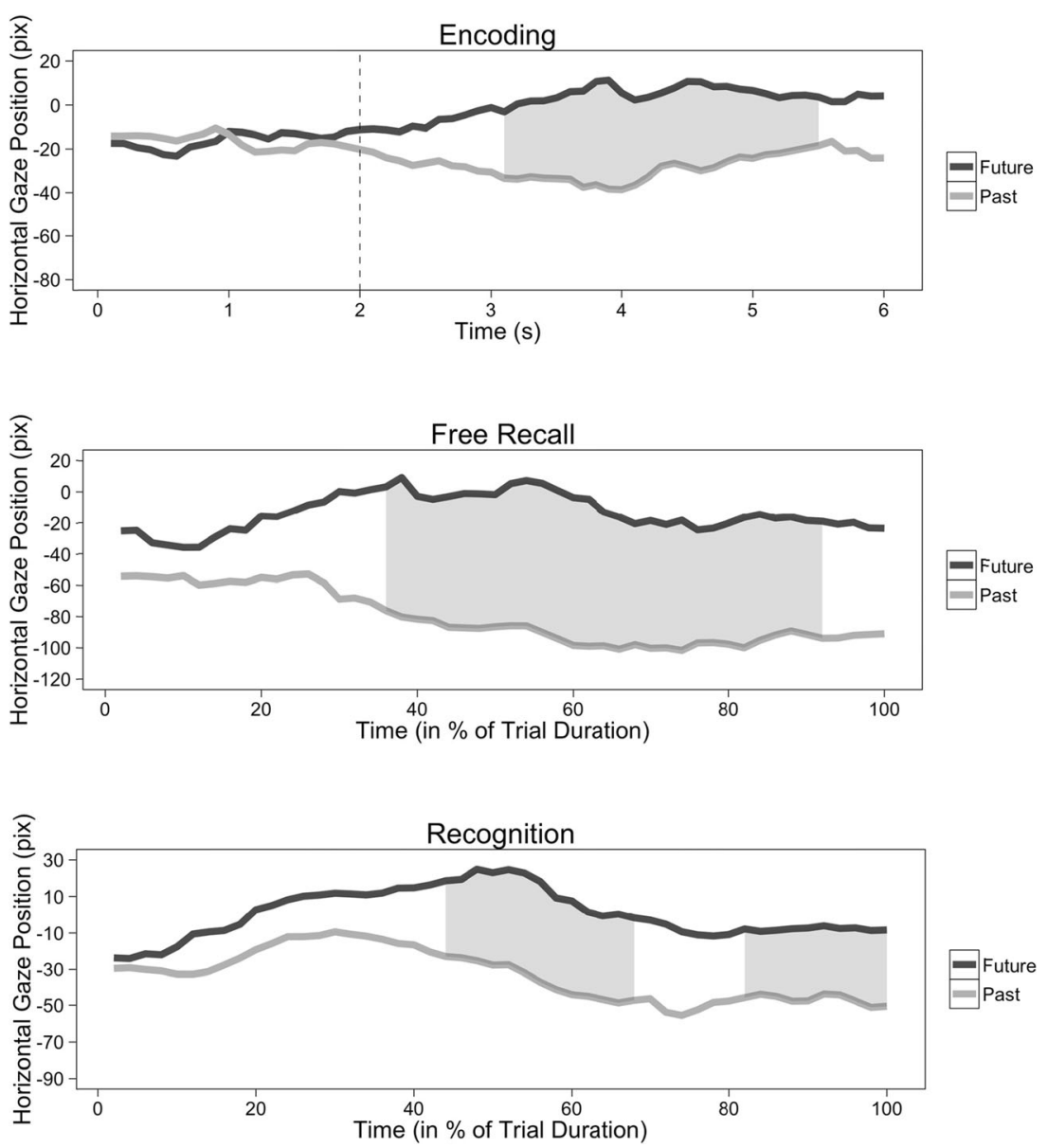

Fig. 2 Mean horizontal eye gaze position (in pixels) for future and past items in the encoding ( $6 \mathrm{~s}$ ), free recall (100 time points), and recognition task (100 time points). Zero at the y-axis represents the middle of the screen. The higher the values, the more to the right the eye position on

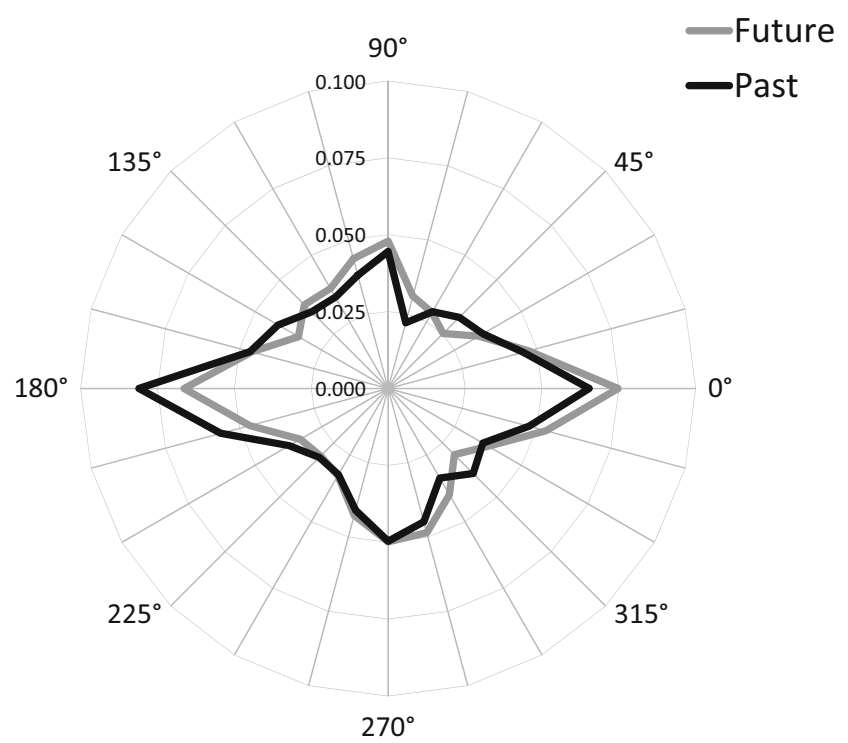

Fig. 3 Distribution of horizontal and vertical changes in eye gaze position in the critical phase of the three tasks the screen. The gray area shows significant differences $(p<.05$ for at least five consecutive time points). The vertical line in the first graph (encoding task) represents word onset (at $2 \mathrm{~s}$ )

followed a mental time line during a mental time travel task (long-term memory without any explicit encoding task), thus indicating that no past-left and future-right association in the encoding is necessary for a mental time line during memory retrieval to exist. We suggest that the results of our study reflect the recruitment of the mental time line, which can influence both encoding and retrieval processes.

The horizontal eye gaze position during encoding significantly predicted the horizontal eye gaze position both during recall and during recognition even when controlling for time (past vs. future). This is a novel finding because previous research in the field of the "blank screen paradigm" usually provided a visual input during encoding. However, when taking the eye gaze position on the vertical axis as dependent variable, the results turned out to be non-significant. One possible explanation for why only the gaze position on the horizontal axis (and not on the vertical axis) predicts the gaze position during retrieval might be that the temporal information that needs to be memorized and retrieved activates a horizontal mental time line while the variance along the vertical dimension is lower and less systematic and therefore not predictive. 
The time-course analysis helps to better understand the mental time line effect that we found. During encoding, the horizontal eye gaze position starts to be shifted to the left with past-related items immediately after the 1-s tone. However, the difference in the horizontal eye gaze position between past and future-related items becomes significant only $1 \mathrm{~s}$ after word onset (see Fig. 2). The time course of the encoding phase suggests that the mental time line not only is implicated in the abstract concept of time (past vs. future as triggered by the tone) but especially in the concrete encoding and assignment of the items to the past versus future categories. In the free recall and recognition tasks, the differences become significant around the middle of the trial, which corresponds roughly to the time point when the items are recalled and recognized. The fact that the differences between past and future-related items were not exclusively present at the end of the trial indicates that the differences were not only driven by the explicit temporal categorization task, which took place at the end of the trial. Thus, the horizontal mental time line is not an epiphenomenon of the explicit temporal categorization (for a discussion see Hartmann et al. 2014), but seems to be essential in the organization (i.e., encoding and retrieval) of knowledge that includes temporal information.

It is important to note that even though participants shifted their gaze more to the left with past items than with future items, in absolute terms (screen coordinates) the gaze position for future items also was on the left part of the screen. This finding can be interpreted as manifestation of pseudo-neglect, i.e., a general tendency to shift the spatial attention slightly to the left in neurologically normal individuals (e.g., Jewel \& McCourt, 2000). Indeed, when averaging the mean gaze position for each participants over the entire experiment, there was a general tendency to orient to the left of the screen center, $M=$ $-24.77, S D=62.20, t(26)=2.069, p=0.049$ (one-sample t-test; 0 is the middle of the screen). A further explanation of the general leftward bias in eye position is reading habit (here left-to-right readers). Indeed it has been shown that the culture influences direction of eye movements for example by using a classical line bisection task and a cancellation task (Rinaldi, Di Luca, Henik, \& Girelli, 2014) or a target-finding task (Smith, Szelest, Friedrich, \& Elias, 2015).

The findings of the study presented here are in agreement with research investigating the mental number line (e.g., Dehaene, Bossini, \& Giraux, 1993): Eye movements revealed right-uplarge and left-down-small spatial-numerical associations (Hartmann et al., 2015, 2016). Together, these results are evidence for the involvement of mental number and time lines, reflected in horizontal (and vertical) eye movements. In our study we found no past versus future effect on the vertical position of the eyes. We did however find also a vertical shift in gaze position when participants thought about their subjective past versus future (Hartmann et al., 2014), or when they processed past and future-related language (Stocker et al., 2015). See Stocker et al. (2015) for possible interpretations of this "vertical" effect.
In memory research it has been shown that returning the eyes to the previous position can facilitate retrieval of stored information (see for example the review articles of Ferreira, Apel, \& Henderson, 2008; Richardson, Altmann, Spivey, \& Hoover, 2009). We propose that the mental time line assists memory retrieval of past versus future events. This interpretation is in line with the findings of Saj et al. (2014). They found that deficits of the spatial representation (left hemispatial neglect) were reflected in deficits of the temporal representation, thus suggesting that spatial representations support temporal representations. The mental time line may play an organizational role in the representation of knowledge. The question whether eye movements along the mental time line play a functional role for the representation and memory retrieval of temporal information cannot be answered at present. Indeed, the study presented here is of correlational nature, thus we cannot conclude about the causal role of eye movements during retrieval of temporal information. For future research, it would be interesting to manipulate eye position as an independent variable (e.g., fixation vs. free viewing) and evaluate its effects on memory performance. In addition to this, manipulating eye position to the left versus right side of the computer screen could reveal potential facilitation or interference with memory retrieval depending on the spatial-temporal congruency.

Ehrlichman and Micic (2012) argue that non-visual eye movements are used in searching memory, in analogy to visual eye-movements that are used to scan the visual environment. Our results extend this finding by showing the specificity of eye movements with respect to a mental time line. To summarize, during encoding, free recall, and recognition of past versus future-related items, eye movements spontaneously followed the spatial structure of temporal associations. Indeed, eye positions shifted to the right with future items when compared to past items. Thus, eye movements are a powerful online and implicit measure that can reveal the organization of abstract knowledge, such as time and numbers (Hartmann, 2015), which are grounded in space.

Acknowledgments We gratefully acknowledge the help of Mirjam Dönni, Janine Fisher, Aniek Habers, and Markus Lauber with the experimental set up and data collection.

\section{References}

Barsalou, L. W. (2008). Grounded cognition. Annual Review of Psychology, 59, 617-645.

Bonato, M., Zorzi, M., \& Umiltà, C. (2012). When time is space: Evidence for a mental time line. Neuroscience \& Biobehavioral Reviews, 36(10), 2257-2273.

Boroditsky, L. (2000). Metaphoric structuring: Understanding time through spatial metaphors. Cognition, 75(1), 1-28.

Casasanto, D., \& Boroditsky, L. (2008). Time in the mind: Using space to think about time. Cognition, 106(2), 579-593. 
Casasanto, D., \& Jasmin, K. (2012). The hands of time: Temporal gestures in English speakers. Cognitive Linguistics, 23(4), 643.

De Vito, S., \& Della Sala, S. (2011). Predicting the future. Cortex, 47(8), $1018-1022$.

Dehaene, S., Bossini, S., \& Giraux, P. (1993). The mental representation of parity and number magnitude. Journal of Experimental Psychology: General, 122(3), 371-396.

Ehrlichman, H., \& Micic, D. (2012). Why do people move their eyes when they think? Current Directions in Psychological Science, 21(2), 96-100.

Fai, A. H. T., \& Cornelius, P. L. (1996). Approximate F-tests of multiple degree of freedom hypotheses in generalized least squares analyses of unbalanced split-plot experiments. The Journal of Statistical Computation and Simulation, 54(4), 363-378.

Ferreira, F., Apel, J., \& Henderson, J. M. (2008). Taking a new look at looking at nothing. Trends in Cognitive Sciences, 12(11), 405-410.

Flumini, A., \& Santiago, J. (2013). Time (also) flies from left to right... if it is needed! In M. Knauff, M. Pauen, N. Sebanz, \& I. Wachmuz (Eds.), Proceedings of the 35th Annual Conference of the Cognitive Science Society (pp. 2315-2320). Austin: Cognitive Science Society.

Glenberg, A. M., Witt, J. K., \& Metcalfe, J. (2013). From the revolution to embodiment 25 years of cognitive psychology. Perspectives on Psychological Science, 8(5), 573-585.

Hartmann, M. (2015). Numbers in the eye of the beholder: What do eye movements reveal about numerical cognition? Cognitive Processing, $16,245-248$.

Hartmann, M., Martarelli, C. S., Mast, F. W., \& Stocker, K. (2014). Eye movements during mental time travel follow a diagonal line. Consciousness and Cognition, 30, 201-209.

Hartmann, M., Mast, F. W., \& Fischer, M. H. (2015). Spatial biases during mental arithmetic: Evidence from eye movements on a blank screen. Frontiers in Psychology, 6, 12.

Hartmann, M., Mast, F. W., \& Fischer, M. H. (2016). Counting is a spatial process: Evidence from eye movements. Psychological Research, 80, 399-409.

Ishihara, M., Keller, P. E., Rossetti, Y., \& Prinz, W. (2008). Horizontal spatial representations of time: Evidence for the STEARC effect. Cortex, 44(4), $454-461$.

Jewell, G., \& McCourt, M. E. (2000). Pseudoneglect: A review and metaanalysis of performance factors in line bisection tasks. Neuropsychologia, 38(1), 93-110.

Johansson, R., Holsanova, J., Dewhurst, R., \& Holmqvist, K. (2012). Eye movements during scene recollection have a functional role, but they are not reinstatements of those produced during encoding. Journal of Experimental Psychology: Human Perception and Performance, 38(5), 1289.

Johansson, R., Holsanova, J., \& Holmqvist, K. (2006). Pictures and spoken descriptions elicit similar eye movements during mental imagery, both in light and in complete darkness. Cognitive Science, 30(6), 1053.

Johansson, R., \& Johansson, M. (2014). Look here, eye movements play a functional role in memory retrieval. Psychological Science, 25(1), 236 242.

Laeng, B., Bloem, I. M., D'Ascenzo, S., \& Tommasi, L. (2014). Scrutinizing visual images: The role of gaze in mental imagery and memory. Cognition, 131(2), 263-283.

Laeng, B., \& Teodorescu, D.-S. (2002). Eye scanpaths during visual imagery reenact those of perception of the same visual scene. Cognitive Science, 26, 207.

Loetscher, T., Bockisch, C. J., Nicholls, M. E. R., \& Brugger, P. (2010). Eye position predicts what number you have in mind. Current Biology, 20(6), R264.

Martarelli, C. S., Chiquet, S., Laeng, B., \& Mast, F. W. (2016). Using space to represent categories: Insights from gaze position. Psychological Research. doi:10.1007/s00426-016-0781-2
Martarelli, C. S., \& Mast, F. W. (2011). Preschool children's eye-movements during pictorial recall. British Journal of Developmental Psychology, $29,425-436$.

Martarelli, C. S., \& Mast, F. W. (2013). Eye movements during long-term pictorial recall. Psychological Research, 77(3), 303-309.

Ouellet, M., Santiago, J., Funes, M. J., \& Lupiáñez, J. (2010). Thinking about the future moves attention to the right. Journal of Experimental Psychology: Human Perception and Performance, 36(1), 17-24.

Richardson, D. C., Altmann, G. T. M., Spivey, M. J., \& Hoover, M. A. (2009). Much ado about eye movements to nothing: A response to Ferreira et al.: Taking a new look at looking at nothing. Trends in Cognitive Sciences, 13(6), 235-236.

Richardson, D. C., \& Kirkham, N. Z. (2004). Multimodal events and moving locations: Eye movements of adults and 6-month-olds reveal dynamic spatial indexing. Journal of Experimental Psychology: General, 133(1), 46-62.

Richardson, D. C., \& Spivey, M. J. (2000). Representation, space and Hollywood Squares: Looking at things that aren't there anymore. Cognition, 76(3), 269-295.

Rinaldi, L., Brugger, P., Bockisch, C. J., Bertolini, G., \& Girelli, L. (2015). Keeping an eye on serial order: Ocular movements bind space and time. Cognition, 142, 291-298.

Rinaldi, L., Di Luca, S., Henik, A., \& Girelli, L. (2014). Reading direction shifts visuospatial attention: An Interactive Account of attentional biases. Acta Psychologica, 151, 98-105.

Saj, A., Fuhrman, O., Vuilleumier, P., \& Boroditsky, L. (2014). Patients with left spatial neglect also Neglect the "Left Side" of Time. Psychological Science, 25(1), 207-214.

Santiago, J., Lupiáñez, J., Pérez, E., \& Funes, M. J. (2007). Time (also) flies from left to right. Psychonomic Bulletin \& Review, 14(3), 512-516.

Schacter, D. L., Addis, D. R., \& Buckner, R. L. (2008). Episodic simulation of future events: Concepts, data, and applications. Annals of the New York Academy of Sciences, 1124, 39-60.

Scholz, A., Mehlhorn, K., \& Krems, J. F. (2016). Listen up, eye movements play a role in verbal memory retrieval. Psychological Research, 80(1), $149-158$.

Smith, A. K., Szelest, I., Friedrich, T. E., \& Elias, L. J. (2015). Native reading direction influences lateral biases in the perception of shape from shading. Laterality, 20(4), 418-433.

Spivey, M. J., \& Geng, J. J. (2001). Oculomotor mechanisms activated by imagery and memory: Eye movements to absent objects. Psychological Research, 65, 235.

Stocker, K. (2012). The time machine in our mind. Cognitive Science, 36(3), 385-420.

Stocker, K., Hartmann, M., Martarelli, C., \& Mast, F. W. (2015). Eye movements reveal mental scanning through time. Cognitive Science.

Tillman, K., Tulagan, N., \& Barner, D. (2015). Building the mental timeline: Spatial representations of time in preschoolers. Sacramento: Poster session presented at the 37th Annual Conference of the Cognitive Science Society.

Torralbo, A., Santiago, J., \& Lupiáñez, J. (2006). Flexible conceptual projection of time onto spatial frames of reference. Cognitive Science, 30(4), 745-757.

Van Dijck, J. P., \& Fias, W. (2011). A working memory account for spatialnumerical associations. Cognition, 119(1), 114-119.

Wantz, A. L., Martarelli, C. S., \& Mast, F. W. (2016). When looking back to nothing goes back to nothing. Cognitive Processing, 17(1), 105-114.

Weger, U. W., \& Pratt, J. (2008). Time flies like an arrow: Space-time compatibility effects suggest the use of a mental timeline. Psychonomic Bulletin and Review, 15(2), 426-430.

Winter, B., Marghetis, T., \& Matlock, T. (2015). Of magnitudes and metaphors: Explaining cognitive interactions between space, time, and number. Cortex, 64, 209-224. 